\title{
Multiple roles of microRNA-146a in immune responses and hepatocellular carcinoma (Review)
}

\author{
HUIHUI WANG ${ }^{1}$, XUEMEI LI ${ }^{1}$, TAO LI ${ }^{1,2}$, LIANZI WANG ${ }^{1}$, \\ XIAN WU ${ }^{1}$, JIAQING LIU ${ }^{1}$, YUANHONG XU ${ }^{1}$ and WEI WEI ${ }^{2}$ \\ ${ }^{1}$ Department of Clinical Laboratory, The First Affiliated Hospital of Anhui Medical University, Hefei, \\ Anhui 230022; ${ }^{2}$ Key Laboratory of Anti-Inflammatory and Immune Medicine, Ministry of Education, \\ Anhui Collaborative Innovation Center of Anti-Inflammatory and Immune Medicine, \\ Institute of Clinical Pharmacology, Anhui Medical University, Hefei, Anhui 230032, P.R. China
}

Received January 31, 2019; Accepted August 6, 2019

DOI: $10.3892 / \mathrm{ol} .2019 .10862$

\begin{abstract}
MicroRNAs (miRNAs/miRs), consisting of 22 nucleotides of single-stranded RNA, participate in post-transcriptional gene regulation by binding to the 3'-untranslated region (UTR) of mRNAs, repressing their translation and promoting their degradation. Studies have shown that certain miRNAs play a key role in the control of various cellular activities, such as inhibiting inflammation, modulating cell differentiation and suppressing cancer growth. The role of miR-146a in the immune response and in the pathogenesis of hepatocellular carcinoma (HCC) has also been investigated. Although some studies have shown that increased miR-146a levels are associated with HCC, others have revealed that miR-146a suppresses cancer cell proliferation, invasion and metastasis. Toll-like receptor 4 (TLR4) signaling has an important role in regulating innate and adaptive immune responses. In addition, TLR4 is functionally expressed in HCC cells and promotes HCC cell proliferation, which can be regulated by miR-146a. The present review focuses on the recent progress in analyzing the multiple roles of miR-146a in mediating the TLR4 pathway and adaptive immune response. Finally, the function of miR-146a in the pathogenesis of HCC is also discussed.
\end{abstract}

Correspondence to: Professor Tao Li, Department of Clinical Laboratory, The First Affiliated Hospital of Anhui Medical University, 218 Jixi Road, Hefei, Anhui 230022, P.R. China

E-mail: limedical1974@126.com

Professor Wei Wei, Institute of Clinical Pharmacology, Anhui Medical University, 81 Meishan Road, Hefei, Anhui 230032, P.R. China

E-mail: wwei@ahmu.edu.cn

Key words: microRNA-146a, hepatocellular carcinoma, immune response, Toll-like receptor 4 , target, gene polymorphism

\section{Contents}

1. Introduction: MicroRNAs (miRNAs/miRs) and miR-146a

2. Role of miR-146a in the Toll-like receptor 4 (TLR4) signaling pathway

3. Role of miR-146a in the adaptive immune response

4. Role of miR-146a in HCC

5. Conclusions and perspectives

\section{Introduction: MicroRNAs (miRNAs/miRS) and miR-146a}

miRNAs, of 18-22 nucleotides in length have been demonstrated to post-transcriptionally downregulate target mRNA expression by binding to the 3 -untranslated region (UTR) of target mRNA sequences $(1,2)$. Each miRNA can regulate multiple target genes, and several miRNAs can also regulate the same gene $(1,2)$. Thus, several miRNAs can cooperatively control the expression of a single target gene with high precision. In addition, miRNAs are typically highly conserved, which reflects the importance of their various functions $(3,4)$. miRNAs play key roles in diverse biological processes, including cell development and differentiation, signaling pathways, cell proliferation, apoptosis and metabolism (5). Moreover, dysregulation of miRNAs results in a number of diseases, such as viral infections, genetic disorders and several types of cancer, such as breast, pancreatic and gastric cancer (6-9). miR-146a and miR-146b are two members of the miR-146 family. These two miRNAs are expressed in chromosomes 5 and 10, respectively, and exhibit a high level of structural similarity, differing in mature sequence by only two nucleotides at the $3^{\prime}$ end (10). Such small differences in structure indicate that these two miRNAs have similar biological functions (10). For example, only mature forms of miR-146a were found when expression of these miRNAs was stimulated by lipopolysaccharide (LPS) in human monocytes, suggesting different post-transcriptional processing mechanisms for these miRNAs (11).

Studies that aimed to determine whether miRNAs play an important role in the innate immune response to microbial infection initially revealed that miR-146a is nuclear factor $\mathrm{\kappa B}$ 
(NF-кB)-dependent (12). Importantly, miR-146a regulates the innate immune response by binding to the 3'-UTR of the TNF receptor-associated factor 6 (TRAF6) and interleukin IL-1 receptor associated kinase 1 (IRAK1) genes, which encode two key adapter molecules downstream of Toll-like receptors (TLRs) and cytokine receptors (Table I) (12). Another study provided evidence that in addition to acting as a modulator of chronic physiological and pathological responses, miR-146a also regulated acute inflammatory responses in human lung alveolar epithelial cells (13). Perry et al (13) demonstrated that exposure of human lung alveolar epithelial cells to interleukin-1 $\beta$ (IL-1 $\beta$ ) resulted in a rapid time- and concentration-dependent increase in miRNA-146a; to a lesser extent, an increase in miRNA-146b expression was only observed at high IL-1 $\beta$ concentrations. This analysis showed that increased miR-146a expression negatively regulated the release of the proinflammatory chemokines IL- 8 and CCL- 5 by targeting the 3'UTRs of their respective mRNAs (Table I) (13). In addition to the two known miR-146a targets, TRAF6 and IRAK1, Hou et al (14) demonstrated that IL-1 receptor-associated kinase 2 (IRAK2) is another target of miR-146a. And in several types of cancer, such as breast, pancreatic and gastric cancer, miR-146a was found to suppress cancer cell proliferation, invasion and metastasis by repressing the expression of epidermal growth factor receptor (EGFR) through a direct mechanism that involves targeting the 3'-UTR of EGFR mRNA (Table I) (7-9). Furthermore, vesicular stomatitis virus infection resulted in the upregulation of miR-146a expression in mouse macrophages through the TLR-myeloid differentiation factor Myd88-independent TLR, but in a retinoic acid-inducible gene I (RIG-I)-NF- $\kappa \mathrm{B}$-dependent manner by targeting TRAF6, IRAK1 and IRAK2 (Table I) (14).

Based on experiments using pulmonary macrophages, peripheral blood mononuclear cells and serum from patients with lung injury caused by Paraquat poisoning, a dual-luciferase reporter assay demonstrated that the IL-6 mRNA is a direct target of miR-146a. Accordingly, it was suggested that increased expression of IL-6 in patients with lung injury caused by Paraquat poisoning was associated with decreased expression of miR-146a (Table I) (15). Furthermore, studies performed using human papillomavirus (HPV)16 E6/E7-positive keratinocytes identified the histone demethylase KDM2B as a new direct target of miR-146a, and two putative binding sites for miR-146a were identified in its 3'-UTR sequence (Table I) (16). These results revealed that the transcriptional repressor c-MYC mediated the downregulation of miR-146a through the binding sites in the miR-146a promoter, resulting in KDM2B overexpression in HPV-mediated tumorigenesis. Thus, miR-146a-5p may have therapeutic potential to significantly inhibit the proliferation and migration of keratinocytes and cervical cancer cells (16). Two novel targets of miR-146a, cyclooxygenase-2 (COX-2) (17) and 5-lipoxygenase-activating protein (FLAP) (Table I) (18), the functions of which are associated with arachidonic acid metabolism, were also reported. Arachidonic acid can be converted to prostaglandins (PGs) or leukotrienes (LTs) by the enzymatic activities of COX-1, COX-2 or 5-lipoxygenase (5-LO), respectively. FLAP functions with 5-LO to convert arachidonic acid to the intermediate leukotriene B4 (LTB4), one of the most potent LTs. FLAP and LTB4 were found to be upregulated in lung cancer due to a hypermethylated miR-146a promoter, and high LTB4 expression supported a favorable microenvironment for tumor growth and metastasis, leading to low overall survival time in patients with lung adenocarcinoma (18). Similarly, decreased miR-146a expression contributed to the upregulation of COX-2 in lung cancer cells (17). Thus, in lung cancer cells, miR-146a acts as an endogenous dual inhibitor of arachidonic acid metabolism by regulating both $\mathrm{PG}$ and $\mathrm{LT}$ production via direct targeting of the COX-2 and FLAP 3'-UTRs $(17,18)$.

In addition to miRNA, next-generation sequencing revealed that long non-coding RNAs (lncRNAs), a class of regulatory RNAs >200 nucleotides without protein-coding function, play important roles in regulating pre-miRNA splicing, mRNA degradation and epigenetic modification (19). Emerging studies have shown that lncRNAs function as endogenous sponges to regulate miR-146a expression by competitively binding to miR-146a (20-23). For instance, lncRNA HCG18 functions as a competitive endogenous RNA (ceRNA) to upregulate TRAF6 expression by sponging miR-146a in intervertebral disc degeneration (20). One study showed that the lncRNA MALAT1 promoted the pro-inflammatory NF- $\kappa \mathrm{B}$ pathway by targeting miR-146a in LPS-induced acute kidney injury (21). Similarly, the lncRNA NIFK-AS1 suppressed M2 macrophage polarization in endometrial cancer by binding to miR-146a (22), and the lncRNA CHRF downregulated miR-146a in osteoarthritis (23). IncRNAs also inhibited miR-146a expression by inducing methylation of the $\mathrm{CpG}$ island in its promoter, such as IncRNA PVT1 (24). However, no lncRNA was found to regulate the expression of miR-146a in hepatocellular carcinoma (HCC).

\section{Role of miR-146a in the toll-like receptor 4 (TLR4) signaling pathway}

As the first barrier of the body against infectious diseases, TLRs serve as the eyes of natural immunity by monitoring and identifying various pathogen-associated molecular patterns. To date, 10 members of the human TLR family have been found in mammals and humans: TLRs $-1,-2,-4$, $-5,-6$ and -10 are expressed on the surface of cells, whereas TLRs $-3,-7,-8$ and -9 are found intracellularly. There are 12 members of the TLR family (TLR1 to TLR9, and TLR11 to TLR13) in rats (25). Among the TLRs, TLR4 signaling plays an important role in initiating the innate immune response. LPS, the principal component of the outer membrane of Gram-negative bacteria, is a strong stimulator of monocytes and macrophages, involved in innate immunity, and induces the production of a variety of inflammatory mediators, including tumor necrosis factor- $\alpha$ (TNF- $\alpha)$, both in vitro and in vivo. However, TLR4 does not bind to LPS directly; the adaptor protein myeloid differentiation factor 2 (MD-2) binds directly to and recognizes the lipophilic component of LPS (lipid A) (26). The process of TLR4 activation is accomplished through a series of steps in which LPS is bound by different LPS-binding proteins and transferred to the MD-2/TLR4 complex. The LPS-binding protein joins with the LPS monomer from the LPS and delivers it to cluster of differentiation-14 (CD14) proteins, which form the final complex comprising of LPS and MD-2/TLR4 (27). 
Table I. Targets of microRNA-146a.

\begin{tabular}{|c|c|c|c|}
\hline Target gene & Type of cell & Functions & (Refs.) \\
\hline EGFR & Breast, pancreatic and gastric cancer cells & Cell proliferation, invasion and metastasis & $(7-9)$ \\
\hline TRAF6, IRAK1 & $\begin{array}{l}\text { LPS-stimulated monocytes, Th17 cells, } \\
\text { human stellate cells }\end{array}$ & $\begin{array}{l}\text { Innate immunity response, } \mathrm{T} \text { cell-mediated } \\
\text { autoimmunity, anti-fibrotic effect }\end{array}$ & $(12,43,59)$ \\
\hline IL-8, CCL-5 & Lung epithelial alveolar & Acute inflammatory responses & (13) \\
\hline IRAK2 & VSV-infected macrophages & Innate immunity response & (14) \\
\hline IL-6 & Pulmonary macrophages & Paraquat poison & $(15)$ \\
\hline KDM2B & HPV16-positive keratinocytes & Cell proliferation, migration & $(16)$ \\
\hline COX-2, FLAP & Lung adenocarcinoma cells & Cell proliferation, migration & $(17,18)$ \\
\hline FADD & T lymphocytes & Anti-apoptotic effect & (34) \\
\hline Stat1 & Treg cells, Tfh cells, NK/T cells & $\begin{array}{l}\text { Immune homeostasis, limiting the } \\
\text { number of Tfh cells, NK/T cell function }\end{array}$ & $(39,54,55)$ \\
\hline Itch & Th2 cells & Th1/Th17 skewing & $(41)$ \\
\hline ICOS & Tfh cells & Limiting the number of GC B cells & $(44)$ \\
\hline Numb & MZ B cells & MZ B cell differentiation & (49) \\
\hline $\mathrm{CFH}$ & HBV-infected hepatocytes & Hepatitis & $(51)$ \\
\hline HNF $1 \alpha$ & Hepatocytes & Hepatitis, hepatic fibrosis & (57) \\
\hline BRCA1 & HUVECS & Microvascular invasion & $(62)$ \\
\hline HAb18G & Hepatocytes & Cell migration, metastasis & (63) \\
\hline
\end{tabular}

TLR4 identifies ligands in two ways, namely, via the TLR4/MyD88/NF-kB and TLR4/TRIF/IRF3 pathways. In the former pathway, the combination of TLR4 and its ligand forms the MD-2/TLR4 complex that subsequently binds to the Toll/IL-1R homology (TIR) domain structure of MyD88 to activate it. The activated TLR4/MyD88 complexes further stimulate IL-1R-associated kinase 4 (IRAK4). MyD88 recruits IRAK4, and the MyD88-IRAK4 complex recruits the IRAK4 substrate IRAK2 or related IRAK1 (26-28). Based on the crystal structure, the MyD88-IRAK4-IRAK2 complex occurs at a stoichiometry of 6:4:4 (29). Phosphorylated IRAK1 and IRAK2 bind to TRAF6 leading to the activation of inhibitor of nuclear factor $\kappa B$ kinase (IKK). Subsequently, the inhibitor of $\kappa \mathrm{B}(\mathrm{I} \kappa \mathrm{B})$ is phosphorylated by IKK, which triggers ubiquitination and proteolysis and removes $\mathrm{I} \kappa \mathrm{B}$ from $N F-\kappa B$. As a result, $N F-\kappa B$ enters the nucleus and triggers subsequent inflammatory reactions by promoting gene transcription of immune-responsive genes and release of cytokines, such as IL-1 $\beta$, TNF- $\alpha$, IL-6 and miR-146a (26-29). A scheme describing how miR-146a functions in the TLR4 signaling pathway is provided in Fig. 1.

On the other hand, overexpression or inappropriate expression of TLR4 has been implicated in several immune-mediated and inflammatory diseases. A variety of extracellular and intracellular negative feedback pathways are involved in maintaining balanced cellular homeostasis following the activation of TIR receptors. miR-146a was shown to terminate the TLR4 signaling cascade to control the activation of mammalian innate immune responses (12). As early as 2006, miR-146a, miR-132 and miR-155 were reported to be upregulated in human monocytes in response to LPS treatment (12). In one study, 200 miRNAs were analyzed after exposing THP-1 cells of the human acute monocytic leukemia cell line to LPS production, and expression of $\mathrm{miR}-146 \mathrm{a} / \mathrm{b}$, miR-132 and miR-155 was enhanced (12). Further investigation revealed that transcriptional induction of miR-146a by LPS, TNF- $\alpha$ and IL-1 $\beta$ was dependent on NF- $\kappa$ B. Notably, miR-146 was found to play a significant role in controlling TLR and cytokine signaling through a negative feedback regulation loop involving the downregulation of IRAK1 and TRAF6 levels, each a key component involved in amplifying the responses of TLR4 signaling (12).

In addition to functions in THP-1 monocytes, miR-146a was also reported to be upregulated in lymphocytes and lung alveolar epithelial cells through the activation of TLR-mediated $\mathrm{NF}-\kappa \mathrm{B}$ signaling $(13,30)$. Moreover, it is well established that soluble decoy TLRs (sTLRs) are effective in blocking TLR signaling (31). For example, LPS-induced NF- $\kappa \mathrm{B}$ activation and TNF production were inhibited by recombinant sTLR4 expressed by macrophages in vitro. In addition, recent investigation in cells of mammals and fish demonstrated that IRF3 negatively regulates the TLR-mediated NF- $\kappa \mathrm{B}$ signaling pathway by targeting TRIF for ubiquitination and degradation (32). In brief, hosts initiate robust activation of the innate immune system to guard against pathogens, including LPS, and mobilize a variety of extracellular and intracellular 


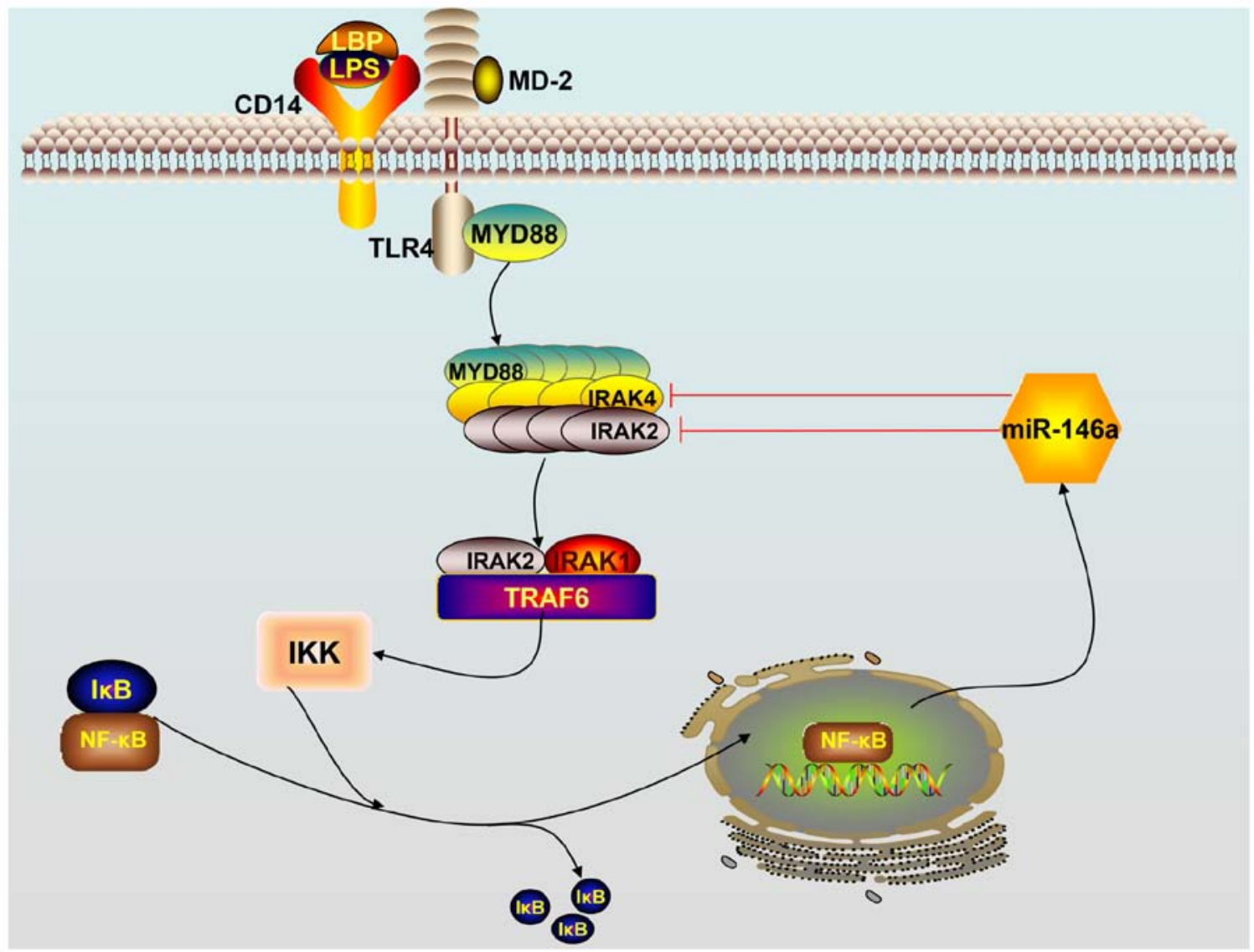

Figure 1. Schematic overview of the TLR4 signaling pathway. Activation of the TLR4 receptor by lipopolysaccharides triggers downstream NF- $\mathrm{B}$ signaling. $\mathrm{NF}-\mathrm{kB}$ enters the nucleus, leading to the expression of miR-146a. Mature miR-146a downregulates IRAK1 and TRAF6 levels by targeting their mRNAs and subsequently terminates the TLR4 signaling pathway cascade. TLR, Toll-like receptor; miR, microRNA; TRAF6, TNF receptor-associated factor 6; IRAK1, interleukin IL-1 receptor-associated kinase 1; NF- $\mathrm{kB}$, nuclear factor $\kappa \mathrm{B}$; LPS, lipopolysaccharides; LBP, lipopolysaccharide binding protein; IKK, inhibitor of

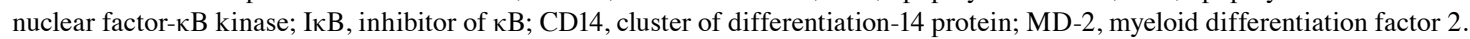

negative feedback pathways to avoid an overstimulated inflammatory state.

\section{Role of miR-146a in the adaptive immune response}

In addition to its expression in monocytes, macrophages that are involved in innate immune responses, miR-146a was also expressed in T cells and B cells, which are associated with adaptive immune responses. During the adaptive immune response, the binding of $\mathrm{T}$ cell receptors (TCRs) to an antigen results in the activation of three main transcription factors, activator protein 1 (AP-1), NF- $\kappa \mathrm{B}$ and NFAT, which are involved in the secretion of early cytokines, particularly IL-2 (33). After the body has defeated the invading organisms, activated $\mathrm{T}$ lymphocytes must be removed in a timely manner to terminate the immune response; this is accomplished by killing the cells that may have developed self-recognition and that are capable of producing reactions to autoantigens (33). miR-146a has a crucial role in accumulating or maintaining the memory T cell pool (34). Evidence that miR-146a is present at a low level in naïve human T cells and is abundantly expressed in human memory $\mathrm{T}$ cells suggests that miR-146a is upregulated during $\mathrm{T}$ cell differentiation (34). miR-146a expression is also induced following $\mathrm{T}$ cell activation, upon $\mathrm{T}$ cell receptor stimulation. A study found that miR-146a impairs AP-1 activity, halts the IL-2 signal to modulate T lymphocyte differentiation and functions as an antiapoptotic factor to protect T lymphocytes from Fas-mediated apoptosis by targeting the Fas-associated via death domain mRNA (Table I) (34). As well as acting as an antiapoptotic factor, miR-146a functions as a $\mathrm{T}$ cell-autonomous factor that controls T-cell-mediated autoimmune responses by targeting TRAF6 and IRAK1 (35). Similar to innate immunity, adaptive immunity (particularly T-cell responses) must be accurately modulated to effectively protect the body and prevent inflammatory diseases, including autoimmune diseases (33-35). Thus, miR-146a is an indispensable modulator in adaptive immunity.

$\mathrm{CD} 4^{+} \mathrm{T}$ cells play a pivotal role in orchestrating the reactions of the innate and adaptive immune systems (36). Following activation, $\mathrm{CD}^{+} \mathrm{T}$ cells can differentiate into distinct subsets of effector T helper (Th) cells, including Th1, Th2, Th9, Th17, $\mathrm{Th} 22$, regulatory $\mathrm{T}$ cells (Tregs) and $\mathrm{T}$ follicular helper cells (Tfh), which have distinct functions in the immune system and regulate appropriate cellular and humoral immune responses to various pathogens (37). Th1 cells produce IL-2, interferon- $\gamma$ $(\mathrm{IFN}-\gamma)$ and TNF- $\beta$, which protect the host against intracellular pathogens; Th2 cells produce IL-4, IL-5, IL-6 and IL-13, and stimulate B cells to proliferate and produce $\mathrm{IgE}$, which is associated with the humoral immune response and allergic inflammation (37). Th17 cells produce the IL-17 family 
proinflammatory cytokines, which are involved in eliminating certain fungi and extracellular pathogens (38). Thus, tightly regulated differentiation of Th cells is vital for providing an effective immune response and avoiding unwanted autoimmunity. Indeed, miR-146a has emerged as a critical modulator of effector $\mathrm{T}$ helper cell differentiation. In Tregs, miR-146a suppresses Th1 responses and controls Treg-maintained immune homeostasis by downregulating the expression of Stat1, a key transcription factor for Th1 effector cell differentiation (Table I) (39). Luo et al (40) revealed that epithelial cell-derived miR-146a induced the expression of IL-10 in monocytes and the inducible IL- $10^{+}$monocytes are capable of suppressing the activities of $\mathrm{CD}^{+}{ }^{+}$effector $\mathrm{T}$ cells and skewing Th2 polarization to avoid allergic reactions in a mouse model of allergic rhinitis. Moreover, miR-146a also prevented aggressive Th1/17 skewing in Th2 cells by targeting Itch, which regulates the production of cytokines (Table I) (41). Interestingly, it was recently reported that miR-146a may be associated with the imbalance of Th1/Th2 differentiation upon decreased IL-4 and increased IFN- $\gamma$ levels, leading to a Th1-driven immune response following acute exposure to airborne particulate matter PM2.5 (42). However, further experiments are needed to verify this hypothesis. In Th17 cells, miR-146a blocked the autocrine IL-6- and IL-21-induced Th17 differentiation pathways in autoreactive $\mathrm{CD}^{+} \mathrm{T}$ cells by targeting TRAF6 and IRAK1 to control T-cell-mediated autoimmunity, such as autoimmune encephalomyelitis (Table I) (43).

miR-146a also serves as a post-transcriptional brake to prevent the accumulation of Tfh cells and germinal center (GC) B-cells, which is crucial for optimal GC B-cell selection $(44,45)$. Moreover, miR-146a-mediated repression of STAT1 contributed to limiting the number of Tfh cells and miR-146a-mediated suppression of inducible $\mathrm{T}$ cell costimulator (ICOS) on Tfh cells, and the corresponding decreased expression of ICOSL on GC B cells contributed to limiting the number of GC B cells (Table I) (44). A recent study highlighted the crucial role of miR-146b, another member of the miR-146 family, in regulating the GC reaction orchestrated by B cells and Tfh cells (46). Similar to previous reports, it was found that the loss of miR-146a in B cells alone is sufficient to cause enhanced GC responses and spontaneous autoimmunity $(44,45)$. However, the inconsistency was that specific deletion of miR-146a in $\mathrm{T}$ cells did not alter the number of Tfh cells, although specific deletion of both miR-146a and miR-146b in T cells increased Tfh cell numbers and elevated GC responses (46). Thus, further studies are needed to explain the intricate molecular mechanism of the miR-146 family in Tfh cells. Although miR-146a was identified as a critical negative regulator of immune reactions, miR-146a overexpression led to enlargement of the spleen and lymph node, inflammatory infiltration in the liver and lung, increased levels of $\mathrm{T}$ cells in peripheral blood and imbalanced homeostasis, and abnormal development of T cells $(47,48)$. Therefore, superabundant or insufficient miR-146 expression is harmful to the maintenance of immune homeostasis. Regardless, the use of miR-146 for the treatment of diseases is challenged by the determination of the optimum dosage.

Despite a good understanding of the role of miR-146a in myeloid and T-cell subsets, its role in B cells is not well understood. Recently, King et al (49) observed an increase in preceding transitional B-cell stages, intact splenic retention and decreased marginal zone (MZ) B cells in miR-146a-deficient mice. Thus, it was speculated that MZ B-cell differentiation was disrupted due to decreased Notch2 signaling and increased Numb expression, with the latter serving not only as a negative regulator of the Notch2 pathway, but also as a direct target of miR-146a (Table I) (49). Overall, miR-146a-mediated regulation of the Notch2 pathway is required for the development of MZ B cells.

\section{Role of miR-146a in HCC}

HCC, the most common primary liver cancer, is the third leading cause of cancer-associated death in the world, according to statistics reported by the World Health Organization (WHO) in 2006 (50). The etiology of HCC is a complex, multistep and multifactor process involving a number of factors, such as chronic infection with hepatitis $\mathrm{B}$ virus (HBV) or hepatitis $\mathrm{C}$ virus (HCV), liver cirrhosis, habitual alcohol abuse and aflatoxin B1 exposure (50). Recently, miR-146a was reported to participate in a variety of pathogenic pathways associated with hepatocarcinogenesis. HBV infects hepatocytes, but does not directly cause cytopathic changes; indeed, immune responses of the host are the main cause of hepatic injury. Li et al (51) found that the protein $\mathrm{HBV} \mathrm{X}(\mathrm{HBx})$ promoted the expression of miR-146a through the NF- $\mathrm{B}$ signaling pathway in HBV-expressing hepatocytes, which subsequently expressed increased levels of miR-146a, leading to inflammation of the liver via downregulation of the target complement factor $\mathrm{H}$ (CFH), an important negative regulator of the complement alternative pathway (Table I; Fig. 2). In the alternative pathway, $\mathrm{CFH}$ accelerates the transfer of $\mathrm{Bb}$ from $\mathrm{C} 3 \mathrm{~b}$ by competing with $\mathrm{B}$ or $\mathrm{Bb}$ for binding to $\mathrm{C} 3 \mathrm{~b}$, through the inhibition of $\mathrm{C} 3$ convertase $(\mathrm{C} 3 \mathrm{bBb})$ formation. A decreased level of $\mathrm{CFH}$ in hepatocytes was associated with excessive activation of the complement alternative pathway and was a direct cause of liver inflammation (51). Hence, elevated HBx-induced expression of miR-146a led to more severe inhibition of $\mathrm{CFH}$ in hepatocytes, resulting in accumulation of $\mathrm{C} 3 \mathrm{bBb}$ and enhancement of the alternative pathway-mediated cytotoxicity, and the subsequent outcomes of hepatitis, liver fibrosis and HCC (51). Similarly, the level of miR-146a was consistently increased in $\mathrm{HCV}$-infected hepatocyte-like cells and liver tissue from $\mathrm{HCV}$-infected patients (52). Further investigation revealed that persistent $\mathrm{HCV}$ infection induced upregulation of miR-146a through the $\mathrm{NF}-\kappa \mathrm{B}$ signaling pathway and that increased expression of miR-146a was involved in key metabolic pathways in liver cells, including the proteasome, fatty acid metabolism and anaerobic energetic metabolism pathways (52). Moreover, increased expression of miR-146a may weaken the immune responses of hepatocytes and help $\mathrm{HCV}$-infected cells escape immune surveillance (52). In HBV or HCV infections, dysregulation of miR-146a promotes liver inflammation and disease progression, in contrast to the usual role of miR-146a in suppressing cancer growth and inhibiting inflammation $(16-18,51,52)$. The finding that miR-371, miR-373 and miR-543 dramatically decreased caspase-8 (Casp-8) gene expression in HCC, by binding to the Casp- 8 mRNA, supports the pro-necrotic and pro-inflammatory functions of miRNAs (53). Furthermore, Visalli et al (53) identified that 


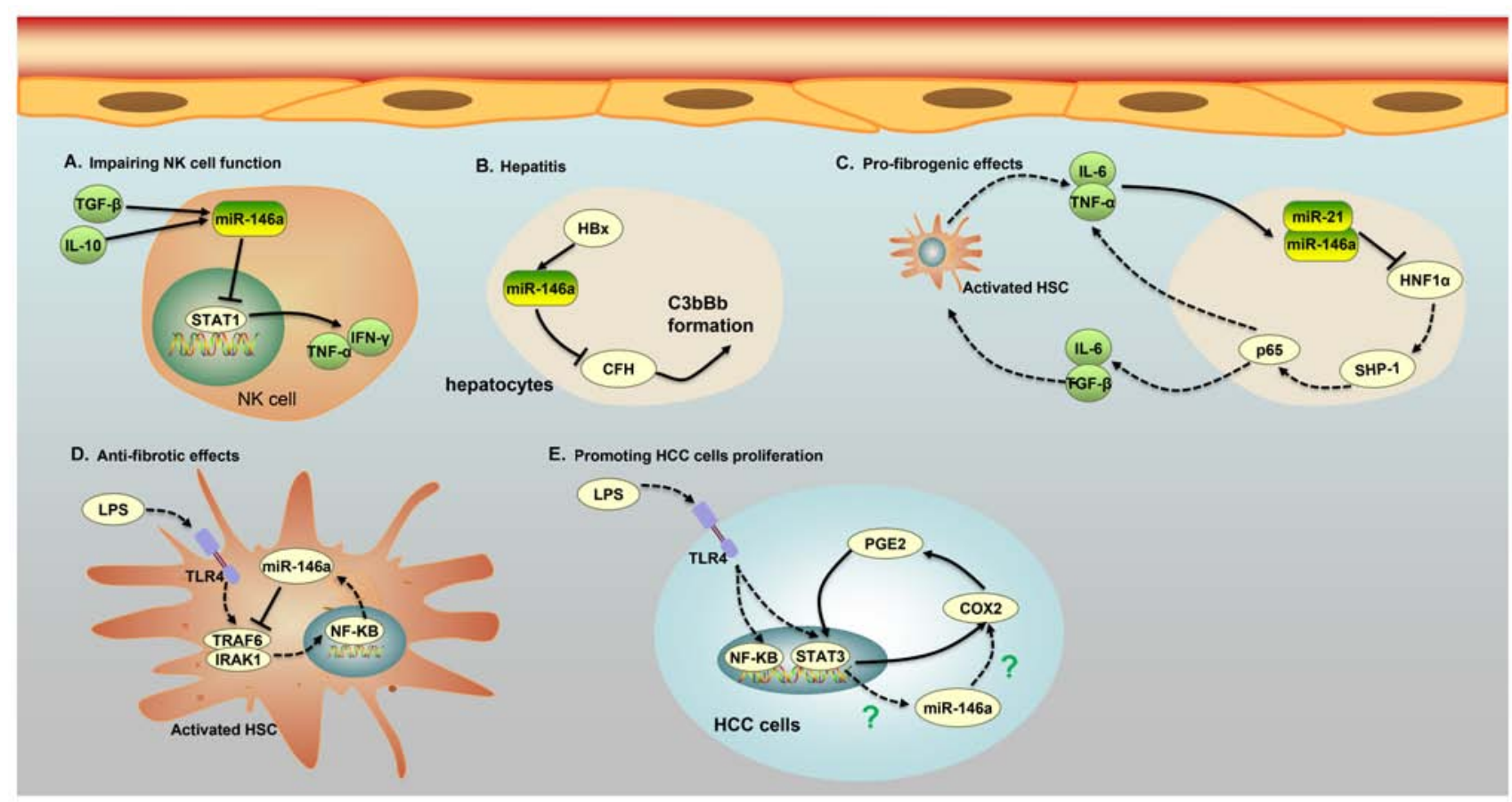

Figure 2. Five major roles of miR-146a in different cells of patients with HCC. (A) miR-146a expression in NK cells of patients with chronic hepatitis B and HCC is induced by IL-10 and TGF- $\beta$, and increased expression of miR-146a weakens the function of NK cells and the secretion of TNF- $\alpha$ and interferon- $\gamma$ by targeting STAT1. (B) The protein HBx promotes the expression of miR-146a, leading to liver inflammation via the downregulation of CFH, an important negative regulator of the complement alternative pathway in $\mathrm{HBV}$-expressing hepatocytes. The low expression of $\mathrm{CFH}$ causes a cascade of $\mathrm{C} 3 \mathrm{bBb}$ formation, which is associated with liver inflammation. (C) An miR-146a-mediated feedback circuit modulates the crosstalk between HSCs and hepatocytes leading to hepatic fibrosis. Inhibition of HNF1 $\alpha$ in hepatocytes by miR- 21 and miR-146a leads to an increase in IL- 6 and TGF- $\beta$ production, which causes the activation of HSCs. On the other hand, activated HSCs secrete IL- 6 and TNF $\alpha$, which further suppress the expression of HNF1 $\alpha$ and SH2 domain-containing phosphatase-1 in hepatocytes. (D) miR-146a can impede the phosphorylation of NF- $\mathrm{KB}$ and suppress the secretion of pro-inflammatory cytokines by binding to IRAK1 and TRAF6 in LPS-induced HSC activation. (E) A COX-2/PGE2/STAT3 positive feedback loop exists in HCC cells, which may involve miR-146a. HCC, hepatocellular carcinoma; IL, interleukin; TGF- $\beta$, transforming growth factor- $\beta$; NK, natural killer; miR, microRNA; TNF, tumor necrosis factor; $\mathrm{CFH}$, target complement factor H; HSC, human stellate cells; HNF, hepatocyte nuclear factor; HBV, hepatitis B virus; HBx, HBV x; LPS, lipopolysaccharide; C3bBb, C3 convertase; NF- $\mathrm{B}$, nuclear factor $\kappa \mathrm{B}$; COX-2, cyclooxygenase-2; PGE2, prostaglandin E2; SHP-1, SH2 domain-containing phosphatase-1; HNF1 $\alpha$, hepatocyte nuclear factor $1 \alpha$; STAT3, signal transducer and activator of transcription 3.

miR-371, miR-373 and miR-543 were overexpressed in HCC, resulting in a significantly decreased level of Casp-8, a marked increase in programmed cell necrosis and an intensive inflammatory process.

Indeed, the level of miR-146a expression was increased not only in $\mathrm{HBV} / \mathrm{HCV}$-infected hepatocytes and liver tissue from $\mathrm{HBV} / \mathrm{HCV}$-infected patients, but also in natural killer (NK) cells and T cells from chronic hepatitis B (CHB) and HCC patients $(54,55)$. miR-146a expression in NK cells of patients with CHB and HCC was induced by IL-10 and TGF- $\beta$, and increased expression of miR-146a weakened the function of NK cells and the secretion of TNF- $\alpha$ and IFN- $\gamma$ by targeting STAT1 (Table I; Fig. 2) (54). Additionally, miR-146a levels were significantly increased in $\mathrm{CD}^{+}$and $\mathrm{CD}^{+} \mathrm{T}$ cells of patients infected with $\mathrm{HBV}$, possibly due to inflammatory cytokines and viral factors, although the details of the underlying mechanisms remain unclear (55). The overexpression of miR-146a in CHB patients impaired T-cell function by targeting STAT1 (55). As a transcription factor, STAT1 plays an important role in antiviral responses by inducing the expression of antiviral IFN-stimulated genes and epitope-specific CTLs. Thus, the upregulation of miR-146a observed in patients with CHB may lead to decreased expression of NK cell-mediated antiviral cytokines and antiviral IFN-stimulated genes, as well as impairment of T/B cell function, promotion of T-cell functional deficiency and immune tolerance to HBV infection $(54,55)$.

Liver fibrosis and cirrhosis are well known to be the main causes of HCC (56). miR-146a was found to be involved in the progression of hepatocellular damage and hepatic fibrogenesis through an inflammatory feedback circuit regulating the crosstalk between hepatocytes and human stellate cells (HSCs) (57). The activation of HSCs into fibroblast-like cells in the injured liver mediated a gradual accumulation of excessive extracellular matrix proteins, leading to hepatic fibrosis (58). In rat models of liver fibrosis, inflammatory cytokines such as IL- 6 and TNF- $\alpha$ were shown to increase the expression of miR-146a and miR-21, which in turn sustained the repression of hepatocyte nuclear factor $1 \alpha(\mathrm{HNF} 1 \alpha)$, a liver-enriched transcription factor that markedly alleviated hepatic fibrosis in hepatocytes. Moreover, HNF1 $\alpha$ suppression in hepatocytes induced phosphorylation of signal transducer and activator of transcription 3 (STAT3) and p65 by regulating the transcriptional expression of $\mathrm{SH} 2$ domain-containing phosphatase-1 (SHP-1), which contributed to IL-6, TNF- $\alpha$ and TGF $\beta 1$ production, thus enhancing hepatocellular inflammation, HSC activation and hepatic fibrosis progression (Table I; Fig. 2) (57). Importantly, another study found that HNF1 $\alpha$ regulated the transcriptional expression of SHP-1 by binding to the SHP-1 promoter in hepatocytes, which was associated with 
activation of the NF- $\mathrm{BB}$ and JAK/STAT pathways, including the phosphorylation of STAT3 and p65 (57). This discovery highlights the central role of miR-146a in liver cell homeostasis and signaling; thus, impairment of miR-146a regulation and signaling may participate in hepatocellular injury and liver cirrhosis. Nonetheless, it was not determined whether HNF1 $\alpha$ binds to the SHP-1 promoter directly or whether other adaptor proteins are required. In addition, it remains to be determined whether other factors are involved in this inflammatory feedback circuit consisting of HNF1 $\alpha$, SHP-1, STAT3, p65, miR-21 and miR-146a. Interestingly, another study revealed the anti-proinflammatory and anti-fibrotic effects of miR-146a on liver injury via the inhibition of LPS/TLR4 signaling (59). TLR4 signaling occurs in activated HSCs, and TLR4 signaling cascades were capable of promoting the secretion of inflammatory cytokines, chemokines and adhesion molecules, which contributed to the progression of liver injury and fibrogenesis (59). Nevertheless, miR-146a downregulated the expression levels of TLR4, IRAK1 and TRAF6, and impeded the phosphorylation of $N F-\kappa B$ to suppress the secretion of pro-inflammatory cytokines and cell proliferation, and to promote apoptosis (Table I; Fig. 2) (59). As most miRNAs exert multiple biological properties due to their multiple modes of action, it was not unexpected that miR-146a had controversial functions in the progression of liver injury and fibrogenesis (1). A single miRNA can target multiple mRNA transcripts, which may be controlled by multiple miRNAs. Thus, it may be reasonable for miR-146a to exhibit different expression characteristics and function in some specific inflammatory settings by downregulating various target genes.

In addition to being expressed in HSCs, TLR4 were found to be functionally expressed in HCC cells upon LPS stimulation, and by activating TLR4 signaling, LPS was able to induce proliferation and clone formation in HCC cells (60). Moreover, positive associations were found among the expression of TLR4, COX-2 and STAT3 in the liver tumor tissues of patients with $\mathrm{HCC}$, and further investigation demonstrated the existence of a COX-2/PGE2/STAT3 positive feedback loop in HCC cells (Fig. 2) (60). Regardless, it was not determined whether miR-146a was involved in the COX-2/PGE2/STAT3 positive feedback loop in HCC cells by LPS stimulation, although miR-146a was demonstrated to be an endogenous dual inhibitor of arachidonic acid metabolism in lung cancer cells by regulating the production of both PG and LT via direct targeting of the COX-2 and FLAP 3' UTRs (17,18). Importantly, another study confirmed that miR-146a expression was regulated by aberrantly activated STAT3 in HCC cells (61). Based on this evidence, it was speculated that miR-146a may be involved in the COX-2/PGE2/STAT3 positive feedback loop in HCC cells through LPS stimulation. Further analysis is needed to verify this speculation and provide a deeper understanding.

miR-146a was also shown to promote the angiogenic activity of endothelial cells in patients with HCC (62). Angiogenesis, a hallmark of cancer, is important in the multistep development of cancer, and endothelial cells (ECs) play a crucial role in the proliferation, migration and arrangement of vascular cavities. By targeting BRCA1, miR-146a upregulated the expression of platelet-derived growth factor receptor $\alpha$ in human umbilical vein endothelial cells, leading to microvascular invasion in patients with HCC (Table I) (62). In contrast to the aforementioned results, Zhang et al (63) demonstrated that miR-146a expression was notably decreased in hepatoma cells and hepatoma tissues due to the methylation of the miR-146a promoter. Furthermore, the recovery of miR-146a was able to prominently inhibit cancer migration, invasion and metastasis by downregulating vascular endothelial growth factor through dual pathways in HCC. miR-146a inhibits nuclear accumulation of $\beta$-catenin by upregulating the expression of adenomatosis polyposis coli (APC), a tumor suppressor, whereas miR-146a downregulates NF- $\kappa \mathrm{B}$ p65 by targeting HAb18G expression (Table I) (63). As a previous study showed that miR-146a directly downregulated the expression of UHRF1, which modulated tumor suppressor gene silencing via DNA methylation (64), it was speculated that the suppression of miR-146a may facilitate self-methylation by upregulating UHRF1 in HCC. However, this possibility was not fully explored and verified, and the molecular mechanism by which miR-146a resulted in upregulation of APC expression requires further investigation.

In addition to the environmental risk factors, genetic factors also have an important function in hepatocellular carcinogenesis. Accumulating studies have provided evidence for a complex link between dysregulated miR-146 expression and HCC development (65-68). A G>C polymorphism (rs2910164) in the stem region opposite the mature miR-146a sequence is caused by a $\mathrm{G}: \mathrm{U}$ to $\mathrm{C}: \mathrm{U}$ mismatch in the stem region of the miR-146a precursor. $\mathrm{Xu}$ et al (65) demonstrated that male individuals carrying the GG genotype of the miR-146a gene had a 2-fold greater susceptibility to HCC than those with the CC genotype; individuals carrying the miR-146a GG genotype also had increased production of mature miR-146a. Jazdzewski et al (69) reported the expression of pre-miR-146a or mature miR-146a from the $\mathrm{C}$ allele to be approximately 2-fold lower than that from the $\mathrm{G}$ allele, with the GC genotype of miR-146a conferring greater susceptibility to an increased risk for papillary thyroid carcinoma compared with the GG or CC genotype. Overall, different etiological factors for papillary thyroid carcinoma and $\mathrm{HCC}$ may account for this discrepancy. Furthermore, a large number of meta-analyses based on several cases showed that the miR-146a rs2910164 polymorphism contributed to increased susceptibility to the development of HCC (66-68). Thus, the genetic variants of miR-146a may be part of a spectrum of genes involved in the etiology of HCC.

Orthotopic liver transplantation is considered as the best choice for patients with end-stage liver disease, whereas acute rejection (AR) of transplants represents the most difficult problem to solve (70). Moreover, it was reported that plasma miRNAs, such as miR-122, miR-192 and miR-146a, can be used as potential diagnostic biomarkers for acute rejection following liver transplantation; as plasma miR-122 and miR-192 indicate liver injury and miR-146a may reflect cellular rejection (71). The association between miR-146a and AR is not surprising as AR is mainly mediated through T-cell-dependent immune pathways, and miR-146a plays a significant role in the control of T-lymphocyte maturation and activation. Surprisingly, increased expression of miR-146a may be associated with multidrug resistance in drug-resistant HCC (72). 


\section{Conclusions and perspectives}

Cumulative evidence has demonstrated that miR-146a serves as a key modulator of innate and adaptive immune responses. However, the mechanism by which miR-146a directly or indirectly affects T-cell differentiation remains controversial, and further studies are required to address this. A number of studies have demonstrated the utility of microRNAs as cancer-associated biomarkers, supported by the finding that some microRNAs displayed altered expression profiles in cancer compared with those in normal tissues. In several types of cancer, such as breast, pancreatic and gastric cancer, miR-146a was found to suppress cancer cell proliferation, invasion and metastasis by repressing the expression of EGFR through a direct mechanism that involves targeting the 3'-UTR of EGFR mRNA. However, an increasing number of studies have demonstrated that miR-146a not only acts as a tumor suppressor, but also functions in hepatocellular oncogenesis, and that genetic variants of miR-146a may be part of a spectrum of genes involved in the etiology of HCC. Several questions remain about the role of miR-146a in oncogenesis. Considering the various roles of miR-146a and its association with HCC, further investigations are required in order to understand the molecular mechanisms of miR-146a-mediated liver disease pathogenesis and HCC development. Finally, the potential of miR-146a as a diagnostic biomarker of liver injury, hepatic fibrosis or HCC requires further investigation.

\section{Acknowledgements}

Not applicable.

\section{Funding}

This study was supported by the Natural Science Foundation of China (grant nos. 81571572, 81201488 and 30801088).

\section{Availability of data and materials}

Not applicable.

\section{Authors' contributions}

HW and XL were major contributors to writing the manuscript. LW and JL produced the tables and figures. HW, XL, LW, JL, $\mathrm{XW}, \mathrm{TL}, \mathrm{YX}$ and $\mathrm{WW}$ checked and revised the manuscript. All authors read and approved the final manuscript.

\section{Ethics approval and consent to participate}

Not applicable.

\section{Patient consent for publication}

Not applicable.

\section{Competing interests}

The authors declare that they have no competing interests.

\section{References}

1. Ambros V: The functions of animal microRNAs. Nature 431: 350-355, 2004.

2. Bartel DP: MicroRNAs: Genomics, biogenesis, mechanism, and function. Cell 116: 281-297, 2004.

3. Lagos-Quintana M, Rauhut R, Lendeckel W and Tuschl T: Identification of novel genes coding for small expressed RNAs. Science 294: 853-858, 2001.

4. Lau NC, Lim LP, Weinstein EG and Bartel DP: An abundant class of tiny RNAs with probable regulatory roles in Caenorhabditis elegans. Science 294: 858-862, 2001.

5. Zhang H, Qu Y, Duan J, Deng T, Liu R, Zhang L, Bai M, Li J, Zhou L, Ning T, et al: Integrated analysis of the miRNA, gene and pathway regulatory network in gastric cancer. Oncol Rep 35: 1135-1146, 2016.

6. Shi XB, Tepper CG and deVere White RW: Cancerous miRNAs and their regulation. Cell Cycle 7: 1529-1538, 2008.

7. Hurst DR, Edmonds MD, Scott GK, Benz CC, Vaidya KS and Welch DR: Breast cancer metastasis suppressor 1 up-regulates miR-146, which suppresses breast cancer metastasis. Cancer Res 69: 1279-1283, 2009.

8. Li Y, Vandenboom TG, Wang Z, Kong D, Ali S, Philip PA and Sarkar FH: miR-146a suppresses invasion of pancreatic cancer cells. Cancer Res 70: 1486-1495, 2010.

9. Kogo R, Mimori K, Tanaka F, Komune S and Mori M: Clinical significance of miR-146a in gastric cancer cases. Clin Cancer Res 17: 4277-4284, 2011.

10. Labbaye C and Testa U: The emerging role of MIR-146A in the control of hematopoiesis, immune function and cancer. J Hematol Oncol 5: 13, 2012.

11. Thomson JM, Newman M, Parker JS, Morin-Kensicki EM, Wright $\mathrm{T}$ and Hammond SM: Extensive post-transcriptional regulation of microRNAs and its implications for cancer. Genes Dev 20: 2202-2207, 2006.

12. Taganov KD, Boldin MP, Chang KJ and Baltimore D: NF-kappaB-dependent induction of microRNA miR-146, an inhibitor targeted to signaling proteins of innate immune responses. Proc Natl Acad Sci USA 103: 12481-12486, 2006.

13. Perry MM, Moschos SA, Williams AE, Shepherd NJ, Larner-Svensson HM and Lindsay MA: Rapid changes in microRNA-146a expression negatively regulate the IL-1beta-induced inflammatory response in human lung alveolar epithelial cells. J Immunol 180: 5689-5698, 2008.

14. Hou J, Wang P, Lin L, Liu X, Ma F, An H, Wang Z and Cao X: MicroRNA-146a feedback inhibits RIG-I-dependent type I IFN production in macrophages by targeting TRAF6, IRAK1 and IRAK2. J Immunol 183: 2150-2158, 2009.

15. $\mathrm{Wu} \mathrm{W}$ and $\mathrm{Li} \mathrm{Y}$ : Lung injury caused by paraquat poisoning results in increased interleukin-6 and decreased microRNA-146a levels. Exp Ther Med 16: 406-412, 2018.

16. Peta E, Sinigaglia A, Masi G, Di Camillo B, Grassi A, Trevisan M, Messa L, Loregian A, Manfrin E, Brunelli M, et al: HPV16 E6 and E7 upregulate the histone lysine demethylase KDM2B through the c-MYC/miR-146a-5p axys. Oncogene 37: 1654-1668, 2018.

17. Cornett AL and Lutz CS: Regulation of COX-2 expression by miR-146a in lung cancer cells. RNA 20: 1419-1430, 2014.

18. Iacona JR, Monteleone NJ and Lutz CS: miR-146a suppresses 5-lipoxygenase activating protein (FLAP) expression and Leukotriene B4 production in lung cancer cells. Oncotarget 9: 26751-26769, 2018

19. Batista PJ and Chang HY: Long noncoding RNAs: Cellular address codes in development and disease. Cell 152: 1298-1307, 2013.

20. Xi Y, Jiang T, Wang W, Yu J, Wang Y, Wu X and He Y: Long non-coding HCG18 promotes intervertebral disc degeneration by sponging miR-146a-5p and regulating TRAF6 expression. Sci Rep 7: 13234, 2017.

21. Ding Y, Guo F, Zhu T, Li J, Gu D, Jiang W, Lu Y and Zhou D: Mechanism of long non-coding RNA MALAT1 in lipopolysaccharide-induced acute kidney injury is mediated by the miR-146a/NF- $\mathrm{BB}$ signaling pathway. Int J Mol Med 41: 446-454, 2018.

22. Zhou YX, Zhao W, Mao LW, Wang YL, Xia LQ, Cao M, Shen J and Chen J: Long non-coding RNA NIFK-AS1 inhibits M2 polarization of macrophages in endometrial cancer through targeting miR-146a. Int J Biochem Cell Biol 104: 25-33, 2018.

23. Yu C, Shi D, Li Z, Wan G and Shi X: Long noncoding RNA CHRF exacerbates IL-6-induced inflammatory damages by downregulating microRNA-146a in ATDC5 cells. J Cell Physiol 234: 21851-21859, 2019. 
24. Liu HT, Fang L, Cheng YX and Sun Q: LncRNA PVT1 regulates prostate cancer cell growth by inducing the methylation of miR-146a. Cancer Med 5: 3512-3519, 2016.

25. Kawai $T$ and Akira $S$ : The role of pattern-recognition receptors in innate immunity: Update on Toll-like receptors. Nat Immunol 11: 373-384, 2010.

26. Shimazu R, Akashi S, Ogata H, Nagai Y, Fukudome K, Miyake K and Kimoto M: MD-2, a molecule that confers lipopolysaccharide responsiveness on Toll-like receptor 4. J Exp Med 189: 1777-1782, 1999.

27. Meng J, Lien E and Golenbock DT: MD-2-mediated ionic interactions between lipid A and TLR4 are essential for receptor activation. J Biol Chem 285: 8695-8702, 2010.

28. Guven-Maiorov E, Keskin O, Gursoy A, VanWaes C, Chen Z, Tsai CJ and Nussinov R: The architecture of the TIR domain signalosome in the toll-like receptor-4 signaling pathway. Sci Rep 5: 13128, 2015.

29. Lin SC, Lo YC and Wu H: Helical assembly in the MyD88-IRAK4-IRAK2 complex in TLR/IL-1R signalling. Nature 465: 885-890, 2010.

30. Cameron JE, Yin Q, Fewell C, Lacey M, McBride J, Wang X, Lin Z, Schaefer BC and Flemington EK: Epstein-Barr virus latent membrane protein 1 induces cellular MicroRNA miR-146a, a modulator of lymphocyte signaling pathways. J Virol 82: 1946-1958, 2008.

31. Iwami KI, Matsuguchi T, Masuda A,Kikuchi T, Musikacharoen T and Yoshikai Y: Cutting edge: Naturally occurring soluble form of mouse Toll-like receptor 4 inhibits lipopolysaccharide signaling. J Immunol 165: 6682-6686, 2000.

32. Zhao $X$, Huo $R$, Yan $X$ and $X u$ T: IRF3 negatively regulates toll-like receptor-mediated NF- $\kappa \mathrm{B}$ signaling by targeting TRIF for degradation in teleost fish. Front Immunol 9: 867, 2018.

33. Schorle H, Holtschke T, Hünig T, Schimpl A and Horak I: Development and function of $\mathrm{T}$ cells in mice rendered interleukin-2 deficient by gene targeting. Nature 352: 621-624, 1991

34. Curtale G, Citarella F, Carissimi C, Goldoni M,Carucci N, Fulci V, Franceschini D, Meloni F, Barnaba V and Macino G: An emerging player in the adaptive immune response: microRNA-146a is a modulator of IL-2 expression and activation-induced cell death in T lymphocytes. Blood 115: 265-273, 2010.

35. Yang L, Boldin MP, Yu Y, Liu CS, Ea CK, Ramakrishnan P, Taganov KD, Zhao JL and Baltimore D: miR-146a controls the resolution of T cell responses in mice. J Exp Med 209: 1655-1670, 2012

36. Zhou L, Chong MM and Littman DR: Plasticity of $\mathrm{CD}^{+} \mathrm{T}$ cell lineage differentiation. Immunity 30: 646-655, 2009.

37. Murphy KM and Stockinger B: Effector T cell plasticity: Flexibility in the face of changing circumstances. Nat Immunol 11: 674-680, 2010.

38. Korn T, Bettelli E, Oukka M and Kuchroo VK: IL-17 and Th17 cells. Annu Rev Immunol 27: 485-517, 2009.

39. Lu LF, Boldin MP, Chaudhry A, Lin LL, Taganov KD, Hanada T, Yoshimura A, Baltimore D and Rudensky AY: Function of miR-146a in controlling Treg cell-mediated regulation of Th1 responses. Cell 142: 914-929, 2010.

40. Luo X, Han M, Liu J, Wang Y, Luo X, Zheng J, Wang S, Liu Z, Liu D, Yang PC and Li H: Epithelial cell-derived micro RNA-146a generates interleukin-10-producing monocytes to inhibit nasal allergy. Sci Rep 5: 15937, 2015.

41. Okoye IS, Czieso S, Ktistaki E, Roderick K, Coomes SM, Pelly VS, Kannan Y, Perez-Lloret J, Zhao JL, Baltimore D, et al: Transcriptomics identified a critical role for Th2 cell-intrinsic miR-155 in mediating allergy and antihelminth immunity. Proc Natl Acad Sci USA 111: E3081-E3090, 2014.

42. Hou T, Liao J, Zhang C, Sun C, Li X and Wang G: Elevated expression of miR-146, miR-139 and miR-340 involved in regulating Th1/Th2 balance with acute exposure of fine particulate matter in mice. Int Immunopharmacol 54: 68-77, 2018.

43. Li B, Wang X, Choi IY, Wang YC, Liu S, Pham AT, Moon H, Smith DJ, Rao DS, Boldin MP and Yang L: miR-146a modulates autoreactive Th17 cell differentiation and regulates organ-specific autoimmunity. J Clin Invest 127: 3702-3716, 2017.

44. Pratama A, Srivastava M, Williams NJ, Papa I, Lee SK, Dinh XT, Hutloff A, Jordan MA, Zhao JL, Casellas R, et al: MicroRNA-146a regulates ICOS-ICOSL signalling to limit accumulation of $\mathrm{T}$ follicular helper cells and germinal centres. Nat Commun 6: 6436, 2015.

45. Boldin MP, Taganov KD, Rao DS, Yang L, Zhao JL, Kalwani M, Garcia-Flores Y, Luong M, Devrekanli A, Xu J, et al: miR-146a is a significant brake on autoimmunity, myeloproliferation and cancer in mice. J Exp Med 208: 1189-1201, 2011.
46. Cho S, Lee HM, Yu IS, Choi YS, Huang HY, Hashemifar SS, Lin LL, Chen MC, Afanasiev ND, Khan AA, et al: Differential cell-intrinsic regulations of germinal center $\mathrm{B}$ and $\mathrm{T}$ cells by miR-146a and miR-146b. Nat Commun 9: 2757, 2018.

47. Guo Q, Zhang J, Li J, Zou L, Zhang J, Xie Z, Fu X, Jiang S, Chen G, Jia Q, et al: Forced miR-146a expression causes autoimmune lymphoproliferative syndrome in mice via downregulation of Fas in germinal center B cells. Blood 121: 4875-4883, 2013.

48. Li Z, Zhang S, Wan Y, Cai M, Wang W, Zhu Y, Li Z, Hu Y, Wang H, Chen H, et al: MicroRNA-146a overexpression impairs the positive selection during T cell development. Front Immunol 8: 2006, 2018.

49. King JK, Ung NM, Paing MH, Contreras JR, Alberti MO, Fernando TR, Zhang K, Pellegrini M and Rao DS: Regulation of marginal Zone B-cell differentiation by MicroRNA-146a. Front Immunol 7: 670, 2017.

50. Mao B and Wang G: MicroRNAs involved with hepatocellular carcinoma (Review). Oncol Rep 34: 2811-2820, 2015.

51. Li JF, Dai XP, Zhang W, Sun SH, Zeng Y, Zhao GY, Kou ZH, Guo Y, Yu H, Du LY, et al: Upregulation of microRNA-146a by hepatitis $\mathrm{B}$ virus $\mathrm{X}$ protein contributes to hepatitis development by downregulating complement factor $\mathrm{H}$. MBio 6: e02459-14, 2015.

52. Bandiera S, Pernot S, El Saghire H, Durand SC, Thumann C, Crouchet E, Ye T, Fofana I, Oudot MA, Barths J, et al: Hepatitis C virus-induced upregulation of MicroRNA miR-146a-5p in hepatocytes promotes viral infection and deregulates metabolic pathways associated with liver disease pathogenesis. J Virol 90: 6387-6400, 2016

53. Visalli M, Bartolotta M, Polito F, Oteri R, Barbera A, Arrigo R, Di Giorgio RM, Navarra G and Aguennouz M: miRNA expression profiling regulates necroptotic cell death in hepatocellular carcinoma. Int J Oncol 53: 771-780, 2018

54. Xu D, Han Q, Hou Z, Zhang C and Zhang J: miR-146a negatively regulates NK cell functions via STAT1 signaling. Cell Mol Immunol 14: 712-720, 2017.

55. Wang S, Zhang X, Ju Y, Zhao B, Yan X, Hu J, Shi L, Yang L, Ma Z, Chen L, et al: MicroRNA-146a feedback suppresses T cell immune function by targeting Stat 1 in patients with chronic hepatitis B. J Immunol 191: 293-301, 2013.

56. Fattovich G, Stroffolini T, Zagni I and Donato F: Hepatocellular carcinoma in cirrhosis: Incidence and risk factors. Gastroenterology 127 (5 Suppl 1): S35-S50, 2004.

57. Qian H, Deng X, Huang ZW, Wei J, Ding CH, Feng RX, Zeng X, Chen YX, Ding J, Qiu L, et al: An HNF1 $\alpha$-regulated feedback circuit modulates hepatic fibrogenesis via the crosstalk between hepatocytes and hepatic stellate cells. Cell Res 25: 930-945, 2015.

58. Weiskirchen R and Tacke F: Cellular and molecular functions of hepatic stellate cells in inflammatory responses and liver immunology. Hepatobiliary Surg Nutr 3: 344-363, 2014.

59. Chen Y, Wu Z, Yuan B, Dong Y, Zhang L and Zeng Z: MicroRNA-146a-5p attenuates irradiation-induced and LPS-induced hepatic stellate cell activation and hepatocyte apoptosis through inhibition of TLR4 pathway. Cell Death Dis 9: $22,2018$.

60. Lin A, Wang G, Zhao H, Zhang Y, Han Q, Zhang C, Tian Z and Zhang J: TLR4 signaling promotes a COX-2/PGE $/$ STAT3 positive feedback loop in hepatocellular carcinoma (HCC) cells. Oncoimmunology 5: e1074376, 2015.

61. Sun X, Zhang J, Hou Z, Han Q, Zhang C and Tian Z: miR-146a is directly regulated by STAT3 in human hepatocellular carcinoma cells and involved in anti-tumor immune suppression. Cell Cycle 14: 243-252, 2015

62. Zhu K, Pan Q, Zhang X, Kong LQ, Fan J, Dai Z, Wang L, Yang XR, Hu J, Wan JL, et al: miR-146a enhances angiogenic activity of endothelial cells in hepatocellular carcinoma by promoting PDGFRA expression. Carcinogenesis 34: 2071-2079, 2013.

63. Zhang Z, Zhang Y, Sun XX, Ma X and Chen ZN: microRNA-146a inhibits cancer metastasis by downregulating VEGF through dual pathways in hepatocellular carcinoma. Mol Cancer 14: 5 , 2015.

64. Zhou L, Zhao X, Han Y, Lu Y, Shang Y, Liu C, Li T, Jin Z, Fan D and Wu K: Regulation of UHRF1 by miR-146a/b modulates gastric cancer invasion and metastasis. FASEB J 27: 4929-4239, 2013.

65. Xu T, Zhu Y, Wei QK, Yuan Y, Zhou F, Ge YY, Yang JR, Su H and Zhuang SM: A functional polymorphism in the miR-146a gene is associated with the risk for hepatocellular carcinoma. Carcinogenesis 29: 2126-2131, 2008. 
66. Peng Q, Li S, Lao X, Chen Z, Li R, Deng Y and Qin X: The association of common functional polymorphisms in mir-146a and mir-196a 2 and hepatocellular carcinoma risk: Evidence from a meta-analysis. Medicine (Baltimore) 93: e252, 2014.

67. Tian T, Wang M, Zhu W, Dai ZM, Lin S, Yang PT, Liu XH, Liu K, Zhu YY, Zheng Y, et al: miR-146a and miR-196a-2 polymorphisms are associated with hepatitis virus-related hepatocellular cancer risk: A meta-analysis. Aging (Albany NY) 9: 381-392, 2017

68. Dong S, Miao AY, Lei W and Chen QW: miR-146a rs2910164 and hepatocellular carcinoma: A meta-analysis. Minerva Med 108: 287-292, 2017.

69. Jazdzewski K, Murray EL, Franssila K, Jarzab B, Schoenberg DR and de la Chapelle A: Common SNP in pre-miR-146a decrease mature miR expression and predisposes to papillary thyroid carcinoma. Proc Natl Acad Sci USA 105: 7269-7274, 2008.
70. Moreno R and Berenguer M: Post-liver transplantation medical complications. Ann Hepatol 5: 77-85, 2006.

71. Hu J, Wang Z, Tan CJ, Liao BY, Zhang X, Xu M, Dai Z, Qiu SJ, Huang XW, Sun J, et al: Plasma microRNA, a potential biomarker for acute rejection after liver transplantation. Transplantation 95: 991-999, 2013.

72. Zhuo L, Liu J, Wang B, Gao M and Huang A: Differential miRNA expression profiles in hepatocellular carcinoma cells and drug-resistant sublines. Oncol Rep 29: 555-562, 2013.

This work is licensed under a Creative Commons Attribution-NonCommercial-NoDerivatives 4.0 International (CC BY-NC-ND 4.0) License. 